\title{
Enantioselective synthesis of isoxazolidinyl nucleosides containing uracil, 5-fluorouracil, thymine and cytosine as new potential anti- HIV drugs
}

\author{
Ugo Chiacchio, ${ }^{* a}$ Antonino Corsaro, ${ }^{a}$ Daniela Iannazzo, ${ }^{b}$ Anna Piperno, ${ }^{b}$ Venerando \\ Pistarà, ${ }^{a}$ Antonio Procopio, ${ }^{\mathrm{d}}$ Antonio Rescifina, ${ }^{\mathrm{a}}$ Giovanni Romeo, ${ }^{* \mathrm{~b}}$ Roberto Romeo, \\ Maria C. R. Siciliano, ${ }^{a}$ and Enza Valveric \\ ${ }^{a}$ Dipartimento di Scienze Chimiche, Università di Catania, Viale Andrea Doria 6, Catania \\ 95125, Italy, ${ }^{b}$ Dipartimento Farmaco-Chimico, Università di Messina, Via SS. Annunziata, \\ Messina 98168, Italy, ${ }^{c}$ Dipartimento di Scienze Microbiologiche, Genetiche e Molecolari, \\ Università di Messina, Salita Sperone 31, Messina 98168, Italy, ${ }^{d}$ Dipartimento di Chimica, \\ Università della Calabria, Arcavacata di Rende, Cosenza 87036, Italy \\ E-mail: uchiacchio@dipchi.unict.it
}

Dedicated to Professor Domenico Spinelli on his $70^{\text {th }}$ birthday

(received 08 Oct 02; accepted 11 Dec 02; published on the web 19 Dec 02)

\begin{abstract}
Two strategies for the enantioselective synthesis of some isoxazolidinyl nucleosides, as potential antiviral drugs, are reported. In particular, a one-step approach based on 1,3-dipolar cycloaddition with vinyl nucleobases and a two-step methodology based on the Vorbrüggen nucleosidation have been exploited in the preparation of 4'-aza-2',3'-dideoxynucleoside analogues containing uracil, 5-fluorouracil, thymine and cytosine.
\end{abstract}

Keywords: Anti-HIV drugs, 1,3-dipolar cycloadditions, isoxazolidines, nucleosides

\section{Introduction}

Derivatives of natural nucleic acids play an important role in current chemotherapy as potent and selective antiviral agents in AIDS therapy. ${ }^{1}$ A series of new compounds, endowed with relevant biological activity, originate from chemical modifications of the nucleic acid fragments at the level of the sugar moiety and/or the heterocyclic base. In this context, the design of novel "ribose" rings has resulted in the discovery of effective biological agents; promising results have been obtained from a new generation of nucleoside analogues where the furanose ring has been replaced by an alternative carbo- or heterocyclic ring. ${ }^{2}$ In particular, isoxazolidinyl nucleosides have been synthesized recently in order to investigate their pharmacological activities. ${ }^{3}$ 


\section{Results and Discussion}

The synthesis of $N, O$-nucleosides $( \pm)-\mathbf{1}$, unsubstituted at the nitrogen atom, has been reported recently. ${ }^{4}$ In particular, $\left( \pm\right.$ )-ADT shows important antiviral and anti-AIDS activity. ${ }^{4}$ However, it is well known that both the enantiomeric purity and absolute configuration are key factors in determining the physiological activity of these molecules.

Accordingly, we have recently investigated the asymmetric version of this reaction route through the use of chiral dipoles: by this route enantiomerically pure ADT and ADF have become accessible (Figure 1). ${ }^{5}$

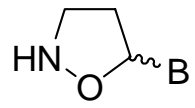

$( \pm)-1$

\begin{tabular}{|l|}
\hline \multicolumn{1}{|c|}{$\mathrm{B}$} \\
\hline Uracil \\
Thymine \\
Cytosine \\
Adenine \\
Guanine \\
\hline
\end{tabular}

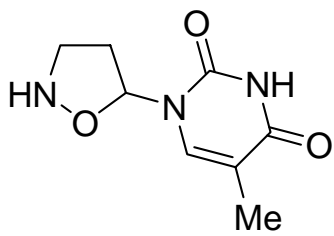

ADT

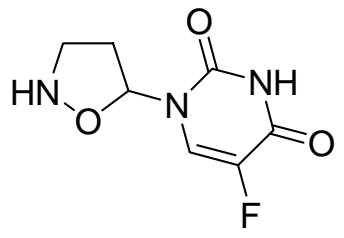

ADF

\section{Figure 1}

As an extension of our ongoing work on the synthesis of isoxazolidinyl analogues having potential antiretroviral effectiveness, ${ }^{6}$ in this paper we exploit the use of a series of vinyl nucleobases in order to prepare 4'-aza-2',3'-dideoxyfuranosyl nucleosides through the 1,3-dipolar cycloaddition methodology. Nitrones containing a chiral auxiliary on the nitrogen atom have been selected as the most convenient precursors for a one-pot reaction pathway towards enantiomerically pure pyrimidine $\mathrm{N}, \mathrm{O}$-nucleosides.

The reaction of ribosyl hydroxylamine $\mathbf{2}$ with formaldehyde and the vinylic bases $\mathbf{4}$ was performed in $\mathrm{CHCl}_{3}$ at $60^{\circ} \mathrm{C}$ for $12 \mathrm{~h}$ to give, through the intermediate unisolated nitrone $\mathbf{3}$, a mixture of two homochiral isoxazolidines 5 and $\mathbf{6}$, epimeric at $\mathrm{C}_{5}$, in a relative ratio 1.5:1 $(40 \%$ yield) (Scheme 1).

The stereochemistry assigned to the obtained adducts is supported by NMR analyses. In fact, NOE measurements performed on $\mathbf{5 a - d}$ and $\mathbf{6 a - d}$ show a positive NOE effect for protons 4" when irradiating 1", thus indicating a cis- relationship between these protons. These data confirm that the sugar moiety has a $\beta$ - configuration in both nucleosides.

Mixtures of diastereomeric invertomers could be observed for compounds 5 and $\mathbf{6}$. Variabletemperature NMR measurements were performed. Upon lowering of the temperature to $-80{ }^{\circ} \mathrm{C}$, it was not possible to reveal the presence of two frozen forms, so suggesting the existence of only one isomer, or a nitrogen inversion sufficiently fast to impart time-averaged properties to the observed compounds.

$\mathrm{PM}^{7}$ quantum-mechanical calculations allow for a clear rationalization of the obtained results. Structural analyses regarding the stabilities of the formed isomers indicate that the $\mathrm{N}_{2}$,$\mathrm{C}_{5}$, trans- isomers are more stable than the cis- derivatives. An energy difference of $4.3 \mathrm{kcal} / \mathrm{mol}$ 
for compound $\mathbf{5 c}$ was calculated in favor of the trans- isomer: this barrier sufficiently explains the experimental fact that only one invertomer was formed. The calculated energy barrier for the nitrogen inversion is $13.9 \mathrm{kcal} / \mathrm{mol}$ : a value of $16.2 \mathrm{kcal} / \mathrm{mol}$ is reported for similar systems. ${ }^{8}$

The marked preference for the trans- form makes these compounds analogues of $\alpha$-nucleosides. Thus, for the $\alpha$ - and $\beta$ - anomers obtained, the difference in configuration of their $\mathrm{C}_{5}$, atoms is compensated by the nitrogen inversion, and both anomers possess the same trans- disposition of their $\mathrm{N}_{2}$,- and $\mathrm{C}_{5}$,- substituents.

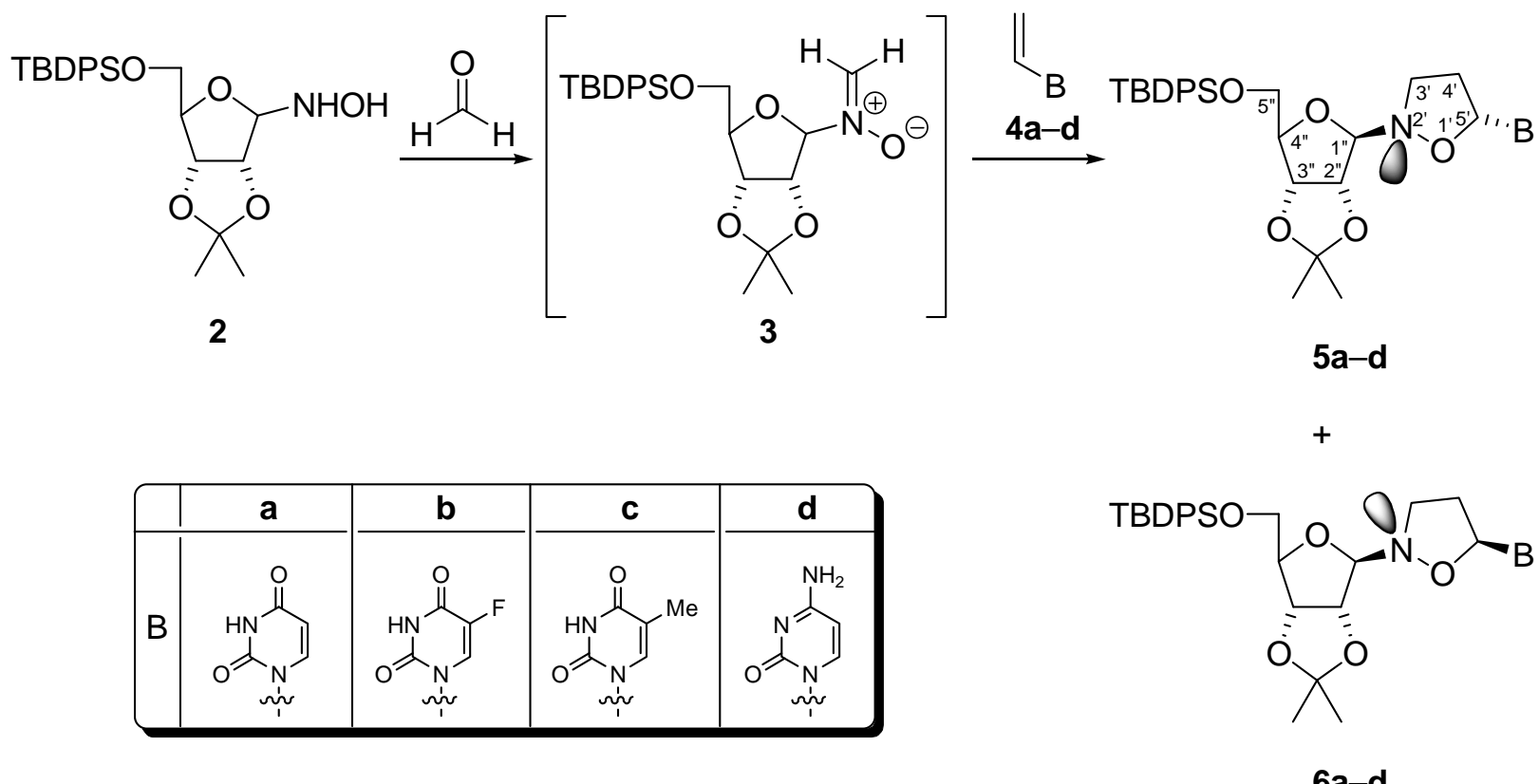

\section{Scheme 1}

On this basis, and according to the quantum-mechanical calculations, the relative configuration at $\mathrm{N}_{2}$, and $\mathrm{C}_{5}$, for nucleosides 5 and $\mathbf{6}$ has been assigned as reported in Scheme 1. The major stereoisomers $\mathbf{5 a}-\mathbf{d}$ can be assigned the configuration $\left(5^{\prime} R\right)$ which is more stable than the configuration $(5 ' S)$ by about $0.8 \mathrm{kcal} / \mathrm{mol}$ : this value is in agreement with the observed $\alpha / \beta$ ratio.

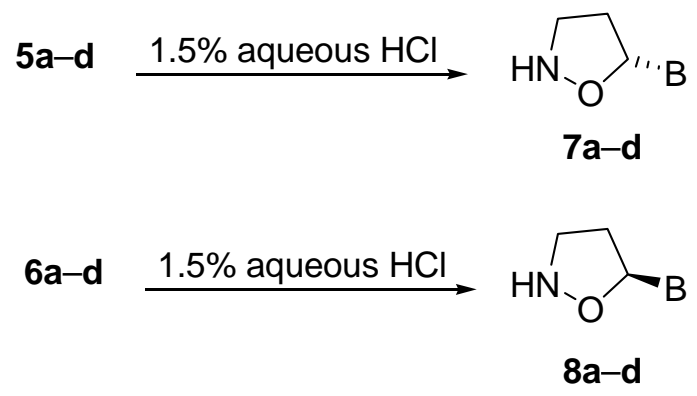

\section{Scheme 2}


The initial goal of the design of a synthetic approach towards the homochiral $\mathrm{N}, \mathrm{O}$ nucleosides $\mathbf{7}$ and $\mathbf{8}$ has been reached by selective cleavage of the sugar moiety, performed by treatment with $1.5 \%$ aqueous $\mathrm{HCl}$ (Scheme 2). Thus, both anomers $7 \mathbf{a}-\mathbf{d}$ and $\mathbf{8 a}-\mathbf{d}$ have been obtained with a global yield of 30\% and 10\% respectively, starting from the nitrone 3 .

Our previously reported synthetic approach to enantiomerically pure isoxazolidinyl nucleosides $\mathbf{7 b}, \mathbf{c}$ and $\mathbf{8 b}, \mathbf{c}$ develops in two steps and includes the cycloaddition of the transient nitrone 3 with vinyl acetate followed by the coupling with silylated nucleobases.

We have compared two procedures: thus, the ribosyl hydroxylamine 2 was reacted with formaldehyde and vinyl acetate to give a mixture of two homochiral isoxazolidines $\mathbf{9}$ and $\mathbf{1 0}$, epimeric at $\mathrm{C}_{5}$, in a relative ratio $1.5: 1(90 \%$ yield $) .{ }^{5}$ The subsequent coupling with silylated uracil 11a, selected as a model compound, in acetonitrile in the presence of TMSOTf at $0^{\circ} \mathrm{C}$, occurs with $80 \%$ yield and affords, in a 1.4:1 ratio, the expected nucleosides $\mathbf{5 a}$ and $\mathbf{6 a}$, which have been separated by flash chromatography and then by HPLC (Scheme 3). The reaction pathway shows a global yield of $72 \%$.

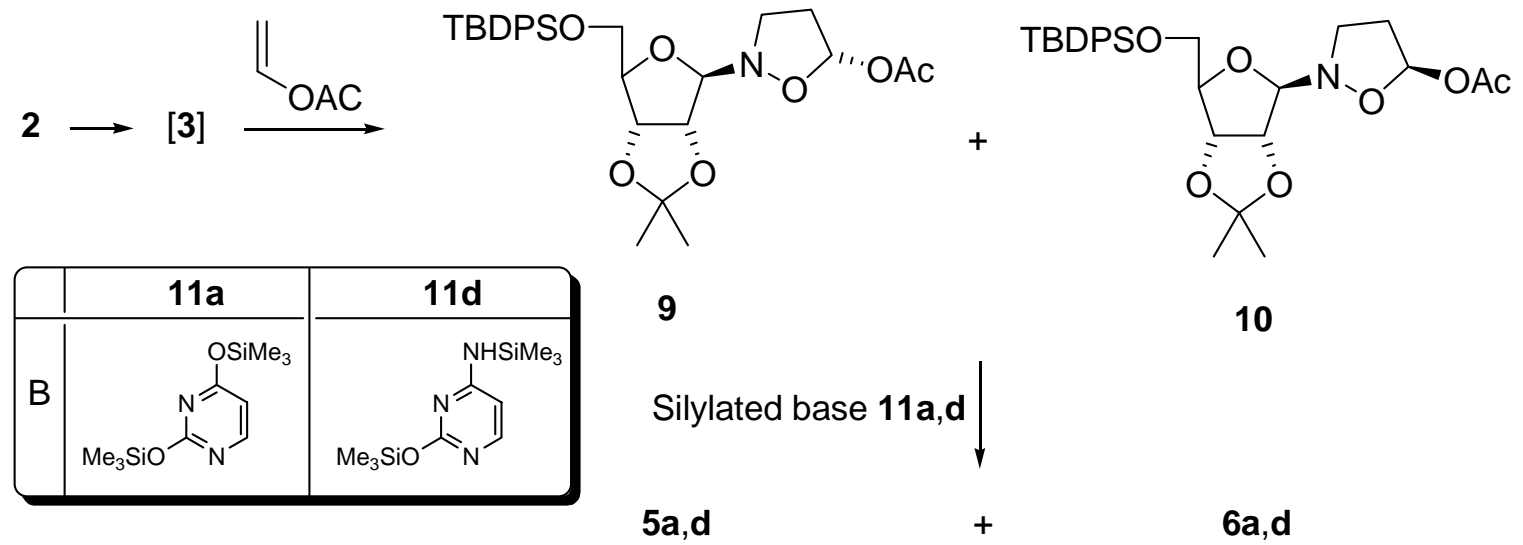

Scheme 3

\section{Conclusions}

Enantiomers of 4'-aza-2',3'-dideoxynucleosides have been prepared by two different synthetic approaches. The results show that the two-step procedure, based on the 1,3-dipolar cycloaddition of the chiral nitrone $\mathbf{4}$ with vinyl acetate and the subsequent Vorbrüggen nucleosidation, leads to clearly better yields.

Biological evaluation of the obtained compounds is in progress. Preliminary data are encouraging: for ADF, tests have been performed on four different cell lines of lymphoid and monocytoid cells. The obtained data indicate that ADF is a good inducer of cell death by apoptosis on Molt-3 cells: at a dose of $128 \mu \mathrm{M}$, this compound causes apoptosis on $50 \%$ of the examined cells. 


\section{Experimental Section}

General Procedures. Melting points were determined with a Kofler apparatus and are uncorrected. Elemental analyses were performed with a Perkin-Elmer elemental analyzer. NMR spectra were recorded on a Varian instrument at 200 or $500 \mathrm{MHz}\left({ }^{1} \mathrm{H}\right)$ and at 50 or $125 \mathrm{MHz}$ $\left({ }^{13} \mathrm{C}\right)$ using deuteriochloroform or deuterated methanol as solvent; chemical shifts are given in ppm from TMS as internal standard. Thin-layer chromatographic separations were performed on Merck silica gel $60-\mathrm{F}_{254}$ precoated aluminum plates. Preparative separations were by columnand flash chromatography using Merck silica gel $0.063-0.200 \mathrm{~mm}$ and $0.035-0.070 \mathrm{~mm}$, respectively, with chloroform-methanol mixtures as eluents. HPLC purifications were made with a preparative column (Microsorb Dynamax 100, $21.4 \times 250 \mathrm{~mm}$ ). The purity of all homochiral compounds has been tested with a Nucleosil Chiral-2, $4 \times 250 \mathrm{~mm}$ column with mixtures of $n$ hexane-2-propanol as eluents.

The identification of samples from different experiments was secured by mixed m.p. and superimposable NMR spectra.

Starting materials. The vinyl-bases $4 \mathbf{a}-\mathbf{d}$ were prepared by literature methods. ${ }^{4}$ Formaldehyde, vinyl acetate, uracil, 5-fluorouracil, thymine, cytosine and D-ribose were purchased from Aldrich Co. All solvents were dried according to literature methods.

Method A. General procedure for 1,3-dipolar cycloaddition reactions between vinyl-bases 4a-d and the Vasella-type nitrone 3

A suspension containing the vinylic base $\mathbf{4 a - d}$ (1 eq.), ribosyl hydroxylamine 2 (1 eq.) and formaldehyde (1 eq.) in chloroform $(10 \mathrm{~mL})$ was heated in a sealed vessel at $60{ }^{\circ} \mathrm{C}$ under stirring, until the hydroxylamine was consumed $(7-8 \mathrm{~h})$. After this time, 0.5 eq. of hydroxylamine and formaldehyde were added and the mixture was left to react for an additional $4 \mathrm{~h}$. Removal of the solvent in vacuum affords a crude material which was purified by flash chromatography to give a mixture of the homochiral isoxazolidines $\mathbf{5 a}-\mathbf{d}$ and $\mathbf{6 a}-\mathbf{d}$, which after separation by HPLC (2-propanol- $n$-hexane) show the physical and spectroscopic data listed below.

Method B. General procedure for the reaction between silylated bases 11a,d and isoxazolidines 9,10

A suspension of the base $\mathbf{5 a , d}(0.62 \mathrm{mmol})$ in dry acetonitrile $(3 \mathrm{~mL})$ was treated with bis(trimethylsilyl)acetamide $(2.54 \mathrm{mmol})$ and heated under reflux for $15 \mathrm{~min}$ with stirring. The clear solution obtained was treated with a solution of the epimeric isoxazolidines 9 or $\mathbf{1 0}^{5}(0.52$ $\mathrm{mmol})$ in dry acetonitrile $(3 \mathrm{~mL})$, trimethylsilyl triflate $(0.78 \mathrm{mmol})$ was added dropwise, and the reaction mixture heated under reflux for $1 \mathrm{~h}$. After cooling at $0{ }^{\circ} \mathrm{C}$, the solution was neutralized by careful addition of aqueous $5 \%$ sodium bicarbonate, and then concentrated in vacuo. After addition of dichloromethane $(8 \mathrm{~mL})$, the organic phase was separated, washed with water $(2 \times$ 
$10 \mathrm{~mL}$ ), dried over sodium sulfate, filtered, and evaporated to dryness. The residue was purified by flash chromatography and then by HPLC (2-propanol- $n$-hexane) to give the homochiral isoxazolidines 5a,d and 6a,d whose physical and spectroscopic data are identical to those previously obtained.

(5'R)-1-\{2-[6-(tert-Butyl-diphenyl-silanyloxymethyl)-2,2-dimethyl-tetrahydro-furo[3,4d][1,3]dioxol-4-yl]-isoxazolidin-5-yl\}-1H-pyrimidine-2,4-dione (5a). Yield 24\% (Method A), $42 \%\left(\right.$ Method B); $[\alpha]_{\mathrm{D}}{ }^{25}=+11.66\left(\mathrm{c} 0.6, \mathrm{CHCl}_{3}\right)$; m.p. $82-84{ }^{\circ} \mathrm{C}$, white solid from ethyl acetate (Anal. Calcd. for $\mathrm{C}_{31} \mathrm{H}_{39} \mathrm{~N}_{3} \mathrm{O}_{7} \mathrm{Si}$ : C, 62.71; H, 6.62; N, 7.08\%. Found: C, 62.54; H, 6.69; N, 7.21\%); $\delta_{\mathrm{H}}\left(\mathrm{CDCl}_{3}, 200 \mathrm{MHz}\right): 1.07(\mathrm{~s}, 9 \mathrm{H}), 1.35$ (s, 3H), $1.53(\mathrm{~s}, 3 \mathrm{H}), 2.17-2.22\left(\mathrm{~m}, 1 \mathrm{H}, \mathrm{H}_{4}{ }^{\prime} \mathrm{b}\right)$, 2.76-2.80 (m, $1 \mathrm{H}, \mathrm{H}_{4}$ ' a), 3.04 (dddd, $1 \mathrm{H}, J=1.5,6.5,8.5,10.0 \mathrm{~Hz}, \mathrm{H}_{3}{ }^{\prime}$ ) $), 3.16$ (dddd, $1 \mathrm{H}, J=2.0$, 5.5, 8.5, $\left.10.5 \mathrm{~Hz}, \mathrm{H}_{3}{ }^{\prime} \mathrm{b}\right), 3.71$ (m, 2H, $\mathrm{H}_{5}$ " $, \mathrm{a}, \mathrm{H}_{5}$ "b), 4.31 (dt, $J=1.5,6 \mathrm{~Hz}, \mathrm{H}_{4}$ "), 4.62 (d, $1 \mathrm{H}, J=$ $1.5 \mathrm{~Hz}, \mathrm{H}_{1}$ "), 4.69-4.72 (m, 2H, H ${ }_{2}$, $\mathrm{H}_{3}$ "), 5.59 (d, J=8.5 Hz, H5), 6.22 (dd, 1H, J = 3.7 Hz, $\mathrm{H}_{5}$ ), 7.37-7.44 (m, 5H, aromatic protons), $7.45\left(\mathrm{~d}, J=8.5 \mathrm{~Hz}, \mathrm{H}_{6}\right), 7.47-7.67(\mathrm{~m}, 5 \mathrm{H}$, aromatic protons), $8.66(\mathrm{bs}, 1 \mathrm{H}, \mathrm{NH}) ; \delta_{\mathrm{C}}\left(\mathrm{CDCl}_{3}, 50 \mathrm{MHz}\right): 25.13,26.80,26.85,36.34,48.67,64.23$, $81.67,83.02,84.30,86.37,100.02,102.22,113.30,127.77,127.81,129.93,132.99,133.06$, $135.50,135.54,139.71,150.00,162.94$.

(5'S)-1-\{2-[6-(tert-Butyl-diphenyl-silanyloxymethyl)-2,2-dimethyl-tetrahydro-furo[3,4d][1,3]dioxol-4-yl]-isoxazolidin-5-yl\}-1H-pyrimidine-2,4-dione (6a). Yield 16\% (Method A), $30 \%\left(\right.$ Method B); $[\alpha]_{\mathrm{D}}{ }^{25}=-29.93\left(\mathrm{c} 0.6, \mathrm{CHCl}_{3}\right)$; m.p. $68-70{ }^{\circ} \mathrm{C}$, white solid from ethyl acetate (Anal. Calcd. for $\mathrm{C}_{31} \mathrm{H}_{39} \mathrm{~N}_{3} \mathrm{O}_{7} \mathrm{Si}$ : C, 62.71; H, 6.62; N, 7.08\%. Found: C, 62.62; H, 6.70; N, 7.16\%); $\delta_{\mathrm{H}}\left(\mathrm{CDCl}_{3}, 200 \mathrm{MHz}\right): 1.07(\mathrm{~s}, 9 \mathrm{H}), 1.34(\mathrm{~s}, 3 \mathrm{H}), 1.54(\mathrm{~s}, 3 \mathrm{H}), 2.19-2.25\left(\mathrm{~m}, 1 \mathrm{H}, \mathrm{H}_{4}{ }^{\prime} \mathrm{b}\right)$,

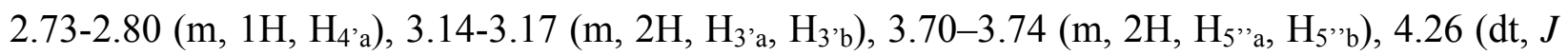
= 3.0, $6.0 \mathrm{~Hz}, \mathrm{H}_{4}$ "), 4.58 (dd, $1 \mathrm{H}, J=4,6.0 \mathrm{~Hz}, \mathrm{H}_{3}$ "), 4.68 (dd, $1 \mathrm{H}, J=2.5,6.5 \mathrm{~Hz}, \mathrm{H}_{2}$ "), 4.81 $\left(\mathrm{d}, 1 \mathrm{H}, J=2.5 \mathrm{~Hz}, \mathrm{H}_{1}\right.$ ") $, 5.61\left(\mathrm{~d}, 1 \mathrm{H}, J=8.5 \mathrm{~Hz}, \mathrm{H}_{5}\right), 5.94\left(\mathrm{dd}, J=3.0,7.5 \mathrm{~Hz}, \mathrm{H}_{5}\right.$ ) $), 7.36-7.46$ (m, 5H, aromatic protons), $7.59\left(\mathrm{~d}, 1 \mathrm{H}, J=8.5 \mathrm{~Hz}, \mathrm{H}_{6}\right), 7.65-7.68(\mathrm{~m}, 5 \mathrm{H}$, aromatic protons), $8.54(\mathrm{bs}, 1 \mathrm{H}, \mathrm{NH}) ; \delta_{\mathrm{C}}\left(\mathrm{CDCl}_{3}, 50 \mathrm{MHz}\right): 25.38,26.78,27.17,37.10,48.15,64.08,81.23,82.77$, $84.33,85.98,99.02,102.16,113.38,127.75,127.77,129.85,129.85,129.91,133.13,133.20$, 135.56, 139.99, 149.91, 163.00 .

(5'R)-1-\{2-[6-(tert-Butyl-diphenyl-silanyloxymethyl)-2,2-dimethyl-tetrahydro-furo-[3,4d][1,3]dioxol-4-yl]-isoxazolidin-5-yl\}-5-fluoro-1H-pyrimidine-2,4-dione (5b). Yield 23\% (Method A), 46\% (Method B). ${ }^{5}$ Physical and spectroscopic data are identical to those reported previously. ${ }^{5}$

(5'S)-1-\{2-[6-(tert-Butyl-diphenyl-silanyloxymethyl)-2,2-dimethyl-tetrahydro-furo-[3,4d][1,3]-dioxol-4-yl]-isoxazolidin-5-yl\}-5-fluoro-1H-pyrimidine-2,4-dione (6b). Yield 17\% (Method A), 26\% (Method B). ${ }^{5}$ Physical and spectroscopic data are identical to those reported previously. 5

(5'R)-1-\{2-[6-(tert-Butyl-diphenyl-silanyloxymethyl)-2,2-dimethyl-tetrahydro-furo[3,4d][1,3]dioxol-4-yl]-isoxazolidin-5-yl\}-5-methyl-1H-pyrimidine-2,4-dione (5c). Yield 25\% (Method A), 42\% (Method B). ${ }^{5}$ Physical and spectroscopic data are identical to those previously reported. $^{5}$ 
(5'S)-1-\{2-[6-(tert-Butyl-diphenyl-silanyloxymethyl)-2,2-dimethyl-tetrahydro-furo[3,4d][1,3]dioxol-4-yl]-isoxazolidin-5-yl\}-5-methyl-1H-pyrimidine-2,4-dione (6c). Yield 15\% (Method A), 30\% (Method B). ${ }^{5}$ Physical and spectroscopic data are identical to those previously reported. $^{5}$

(5'R)-4-Amino-1-\{2-[6-(tert-butyl-diphenyl-silanyloxymethyl)-2,2-dimethyl-tetrahydrofuro-[3,4-d][1,3]-dioxol-4-yl]-isoxazolidin-5-yl\}-1H-pyrimidin-2-one $\quad$ (5d). Yield 22\% (Method A), 44\% (Method B); $[\alpha]_{\mathrm{D}}{ }^{25}=-14.6\left(\mathrm{c} 1.02, \mathrm{CHCl}_{3}\right)$; m.p. $80-82{ }^{\circ} \mathrm{C}$, yellow solid from ethyl acetate (Anal. Calcd. for $\mathrm{C}_{31} \mathrm{H}_{40} \mathrm{~N}_{4} \mathrm{O}_{6} \mathrm{Si}$ : C, 62.81; H, 6.80; N, 9.45\%. Found: C, 62.90; H, 6.75; N, 9.52\%); $\delta_{\mathrm{H}}\left(\mathrm{CDCl}_{3}, 200 \mathrm{MHz}\right): 1.06(\mathrm{~s}, 9 \mathrm{H}), 1.35(\mathrm{~s}, 3 \mathrm{H}), 1.53(\mathrm{~s}, 3 \mathrm{H}), 2.23(\mathrm{dddd}, 1 \mathrm{H}$, $\left.J=1.5,2.5,7.0,13.5 \mathrm{~Hz}, \mathrm{H}_{4^{\prime} \mathrm{a}}\right), 2.79\left(\mathrm{dq}, 1 \mathrm{H}, J=6.0,7.0 \mathrm{~Hz}, \mathrm{H}_{4^{\mathrm{r}} \mathrm{b}}\right), 3.07\left(\mathrm{~m}, 2 \mathrm{H}, \mathrm{H}_{3^{\prime} \mathrm{a}}, \mathrm{H}_{3^{\prime} \mathrm{b}}\right), 3.72$ (ddd, $2 \mathrm{H}, J=6.0,7.0,11.0, \mathrm{~Hz}, \mathrm{H}_{5^{\prime \prime} \mathrm{a},} \mathrm{H}_{5^{\prime \prime}}$ ) $, 4.28\left(\mathrm{dt}, 1 \mathrm{H}, J=1.5,5.5 \mathrm{~Hz}, \mathrm{H}_{4^{\prime \prime}}\right), 4.65(\mathrm{~d}, 1 \mathrm{H}, J=$ $\left.2.0 \mathrm{~Hz}, \mathrm{H}_{1^{\prime \prime}}\right), 4.71\left(\mathrm{~m}, 2 \mathrm{H}, \mathrm{H}_{2^{\prime \prime}}, \mathrm{H}_{3^{\prime \prime}}\right), 5.53\left(\mathrm{~d}, 1 \mathrm{H}, J=7.0 \mathrm{~Hz}, \mathrm{H}_{5}\right), 6.21(\mathrm{dd}, 1 \mathrm{H}, J=2.5,7.0 \mathrm{~Hz}$, $\left.\mathrm{H}_{5^{\prime}}\right), 7.36-7.44\left(\mathrm{~m}, 5 \mathrm{H}\right.$, aromatic protons), $7.56\left(\mathrm{~d}, 1 \mathrm{H}, J=7.0 \mathrm{~Hz}, \mathrm{H}_{6}\right), 7.64-7.66(\mathrm{~m}, 5 \mathrm{H}$, aromatic protons); $\delta_{\mathrm{C}}\left(\mathrm{CDCl}_{3}, 50 \mathrm{MHz}\right): 19.21,25.17,26.79,36.63,47.81,64.29,81.45,82.89$, $85.60,86.20,93.42,99.69,113.25,127.76,129.86,133.08,133.13,135.27,141.14,155.67$, 165.60.

(5'S)-4-Amino-1-\{2-[6-(tert-butyl-diphenyl-silanyloxymethyl)-2,2-dimethyl-tetrahydro-furo[3,4-d][1,3]-dioxol-4-yl]-isoxazolidin-5-yl\}-1H-pyrimidine-2-one (6d). Yield 18\% (Method A), $28 \%\left(\right.$ Method B); $[\alpha]_{\mathrm{D}}^{25}=+4.96\left(\right.$ c $\left.0.3, \mathrm{CHCl}_{3}\right)$; pale yellow oil (Anal. Calcd. for $\mathrm{C}_{31} \mathrm{H}_{40} \mathrm{~N}_{4} \mathrm{O}_{6} \mathrm{Si}: \mathrm{C}, 62.81 ; \mathrm{H}, 6.80 ; \mathrm{N}, 9.45 \%$. Found: $\left.\mathrm{C}, 62.35 ; \mathrm{H}, 6.84 ; \mathrm{N}, 9.52 \%\right) ; \delta_{\mathrm{H}}\left(\mathrm{CDCl}_{3}\right.$, $200 \mathrm{MHz}$ ): 1.06 (s, 9H), 1.35 (s, 3H), 1.54 (s, 3H), 2.24 (dddd, $1 \mathrm{H}, J=3.4,5.7,8.5,13.5 \mathrm{~Hz}$, $\left.\mathrm{H}_{4^{\prime} \mathrm{b}}\right), 2.82\left(\mathrm{~m}, 1 \mathrm{H}, \mathrm{H}_{4^{\prime} \mathrm{a}}\right), 3.09\left(\mathrm{~m}, 2 \mathrm{H}, \mathrm{H}_{3^{\prime} \mathrm{a}}, \mathrm{H}_{3^{\prime} \mathrm{b}}\right), 3.70\left(\mathrm{dd}, 1 \mathrm{H}, J=7.0,10.5 \mathrm{~Hz}, \mathrm{H}_{5^{\prime \prime} \mathrm{a}}\right), 3.74(\mathrm{dd}$, $1 \mathrm{H}, J=5.5,10.5 \mathrm{~Hz}, \mathrm{H}_{5^{\prime \prime} \mathrm{b}}$ ), 4.27 (ddd, $\left.1 \mathrm{H}, J=2.5,6.5,7.0 \mathrm{~Hz}, \mathrm{H}_{4^{\prime \prime}}\right), 4.60$ (dd, $1 \mathrm{H}, J=2.5,6.5$ $\left.\mathrm{Hz}, \mathrm{H}_{3^{\prime \prime}}\right), 4.67\left(\mathrm{dd}, 1 \mathrm{H}, J=2.5,6.0 \mathrm{~Hz}, \mathrm{H}_{2^{\prime \prime}}\right), 4.79\left(\mathrm{~d}, 1 \mathrm{H}, J=2.5 \mathrm{~Hz}, \mathrm{H}_{1^{\prime \prime}}\right), 5.49(\mathrm{~d}, 1 \mathrm{H}, J=7.0$ $\left.\mathrm{Hz}, \mathrm{H}_{5}\right), 5.97\left(\mathrm{dd}, 1 \mathrm{H}, J=3.0,7.0 \mathrm{~Hz}, \mathrm{H}_{5^{\prime}}\right), 7.37-7.44\left(\mathrm{~m}, 5 \mathrm{H}\right.$, aromatic protons, cytosine $\left.\mathrm{H}_{6}\right) ; \delta_{\mathrm{C}}$ $\left(\mathrm{CDCl}_{3}, 50 \mathrm{MHz}\right): 19.22,25.40,26.81,27.15,29.68,37.56,47.99,64.02,81.51,82.77,85.52$, $85.82,93.16,99.54,113.28,127.77,129.86,135.61,141.38,155.42,165.43$.

\section{General procedure for hydrolysis of homochiral isoxazolidines $5 a-d$ and $6 a-d$}

The isoxazolidine $\mathbf{5 a - d}$ or $\mathbf{6 a - d}$ was dissolved in a $1.5 \% \mathrm{HCl}$ solution in $\mathrm{EtOH}(2.5 \mathrm{~mL})$, and the reaction mixture was stirred at room temperature for $3 \mathrm{~h}$. The solution was brought to $\mathrm{pH} 10$ by adding aqueous $10 \%$ sodium carbonate and extracted with dichloromethane $(2 \times 10 \mathrm{~mL})$. The organic phase, dried over sodium sulfate, was filtered and evaporated to dryness. The residue was purified by radial chromatography (chloroform-methanol 9:1) to furnish the homochiral $N, O$-nucleosides $7 \mathbf{a}-\mathbf{d}$ and $\mathbf{8 a}-\mathbf{d}$.

$\left(\mathbf{5}^{\prime} \boldsymbol{R}\right)$-1-Isoxazolidin-5-yl-1H-pyrimidine-2,4-dione (7a). Yield 91\%; $[\alpha]_{\mathrm{D}}^{25}=+97.5$ (c 0.19; $\mathrm{CH}_{3} \mathrm{OH}$ ); m.p. $183-185^{\circ} \mathrm{C}$, white solid from methanol (Anal. Calcd. for $\mathrm{C}_{7} \mathrm{H}_{9} \mathrm{~N}_{3} \mathrm{O}_{3}: \mathrm{C}, 45.90 ; \mathrm{H}$, 4.95; N, 22.94\%. Found: C, 45.92; H, 4.96; N, 22.93\%); $\delta_{\mathrm{H}}\left(\mathrm{CD}_{3} \mathrm{OD}, 200 \mathrm{MHz}\right): 2.62(\mathrm{~m}, 1 \mathrm{H}$, $\left.\mathrm{H}_{2}{ }^{\prime} \mathrm{b}\right), 3.12\left(\mathrm{~m}, 1 \mathrm{H}, \mathrm{H}_{3^{\prime} \mathrm{a}}\right), 3.87\left(\mathrm{~m}, 1 \mathrm{H}, \mathrm{H}_{3^{\prime} \mathrm{b}}\right), 5.82\left(\mathrm{~d}, 1 \mathrm{H}, J=7.5 \mathrm{~Hz}, \mathrm{H}_{5}\right), 6.19(\mathrm{dd}, 1 \mathrm{H}, J=$ 
3.1,7.9 Hz, $\mathrm{H}_{5}$ ), 7.41 (d, $\left.1 \mathrm{H}, J=7.5 \mathrm{~Hz}, \mathrm{H}_{6}\right), 10.28$ (bs, $\left.1 \mathrm{H}, \mathrm{NH}\right) ; \delta_{\mathrm{C}}\left(\mathrm{CD}_{3} \mathrm{OD}, 50 \mathrm{MHz}\right): 30.98$, 47.34, 85.52, 100.80, 102.18, 151.06, 164.22.

(5'S)-1-Isoxazolidin-5-yl-1H-pyrimidine-2,4-dione (8a). Yield 92\%; $[\alpha]_{\mathrm{D}}{ }^{25}=-84.7$ (c 0.23 ; $\mathrm{CH}_{3} \mathrm{OH}$ ); m.p. $183-185^{\circ} \mathrm{C}$, white solid from methanol (Anal. Calcd. for $\mathrm{C}_{7} \mathrm{H}_{9} \mathrm{~N}_{3} \mathrm{O}_{3}: \mathrm{C}, 45.90 ; \mathrm{H}$, 4.95; N, 22.94\%. Found: C, 45.89; H, 4.93; N, 22.91\%).

$\left(5^{\prime} \boldsymbol{R}\right)-5$-Fluoro-1-isoxazolidin-5-yl-1H-pyrimidine-2,4-dione $\mathbf{( 7 b )}$. Yield $90 \% ;[\alpha]_{\mathrm{D}}{ }^{25}=+52.4$ (c 0.43 ; MeOH); m.p. $155-157^{\circ} \mathrm{C}$ (lit. $.^{5} 153-155^{\circ} \mathrm{C}$ ), white solid from methanol (Anal. Calcd. for $\mathrm{C}_{7} \mathrm{H}_{8} \mathrm{FN}_{3} \mathrm{O}_{3}$ : C, 41.80; H, 4.01; N, 20.89\%. Found: C, 41.82; H, 3.99; N, 20.92\%). Physical and spectroscopic data are identical to those reported previously. ${ }^{5}$

(5'S)-5-Fluoro-1-isoxazolidin-5-yl-1H-pyrimidine-2,4-dione (8b). Yield 89\%; $[\alpha]_{\mathrm{D}}{ }^{25}=-63.8$ (c 0.17; MeOH); white solid from methanol, m.p. $155-157{ }^{\circ} \mathrm{C}$ (lit. ${ }^{5} 153-155{ }^{\circ} \mathrm{C}$ ); (Anal. Calcd. for $\mathrm{C}_{7} \mathrm{H}_{8} \mathrm{FN}_{3} \mathrm{O}_{3}$ : C, 41.80; H, 4.01; N, 20.89\%. Found: C, 41.79; H, 4.03; N, 20.87\%). Physical and spectroscopic data are identical to those reported previously. ${ }^{5}$

$\left(5^{\prime} R\right)$-1-Isoxazolidin-5-yl-5-methyl-1H-pyrimidine-2,4-dione (7c). Yield $93 \%$; $[\alpha]_{\mathrm{D}}{ }^{25}=-82.7$ (c 0.21 ; MeOH); m.p. 201-203 ${ }^{\circ} \mathrm{C}$, white solid from methanol (lit. ${ }^{5}$ 202-204 ${ }^{\circ} \mathrm{C}$ ), (Anal. Calcd. for $\mathrm{C}_{8} \mathrm{H}_{11} \mathrm{~N}_{3} \mathrm{O}_{3}$ : C, 48.73; H, 5.62; N, 21.31\%. Found: C, 48.74; H, 5.63; N, 21.32\%). Physical and spectroscopic data are identical to those reported previously. ${ }^{5}$

(5'S)-1-Isoxazolidin-5-yl-5-methyl-1H-pyrimidine-2,4-dione (8c). Yield 91\%; $[\alpha]_{\mathrm{D}}{ }^{25}=+94.3$ (c 0.14; MeOH); m.p. 201-203 ${ }^{\circ} \mathrm{C}$, white solid from methanol (lit. ${ }^{5}$ 202-204 ${ }^{\circ} \mathrm{C}$ ); (Anal. Calcd. for $\mathrm{C}_{8} \mathrm{H}_{11} \mathrm{~N}_{3} \mathrm{O}_{3}$ : C, 48.73; H, 5.62; N, 21.31\%. Found: C, 48.71; H, 5.60; N, 21.33\%). Physical and spectroscopic data are identical to those reported previously. ${ }^{5}$

(5'S)-4-Amino-1-isoxazolidin-5-yl-1H-pyrimidin-2-one (7d). Yield 94\%; $[\alpha]_{\mathrm{D}}{ }^{25}=-12.7$ (c 0.3; $\mathrm{MeOH}$ ); m.p. $190-194{ }^{\circ} \mathrm{C}$, amorphous solid (Anal. Calcd. for $\mathrm{C}_{7} \mathrm{H}_{10} \mathrm{~N}_{4} \mathrm{O}_{2}: \mathrm{C}, 46.15 ; \mathrm{H}, 5.53 ; \mathrm{N}$, 30.75\%. Found: C, 46.70; H, 5.57; N, 31.02\%); $\delta_{\mathrm{H}}\left(\mathrm{CD}_{3} \mathrm{OD}, 200 \mathrm{MHz}\right): 2.39-2.54\left(\mathrm{~m}, 1 \mathrm{H}, \mathrm{H}_{4^{\prime} \mathrm{a}}\right)$, 2.57-2.71 (m, 1H, $\left.\mathrm{H}_{4^{\prime} \mathrm{b}}\right), 3.11-3.34\left(\mathrm{~m}, 2 \mathrm{H}, \mathrm{H}_{3^{\prime} \mathrm{a}}, \mathrm{H}_{3^{\prime} \mathrm{b}}\right), 5.89\left(\mathrm{~d}, 1 \mathrm{H}, J=7.4 \mathrm{~Hz}, \mathrm{H}_{5}\right), 5.98(\mathrm{dd}, J=$ 3.6, 7.2 Hz, $\left.\mathrm{H}_{5^{\prime}}\right), 7.63\left(\mathrm{~d}, 1 \mathrm{H}, J=7.4 \mathrm{~Hz}, \mathrm{H}_{6}\right) ; \delta_{\mathrm{C}}\left(\mathrm{CD}_{3} \mathrm{OD}, 50 \mathrm{MHz}\right): 37.42,49.52,90.96,95.89$, $143.75,158.34,167.37$.

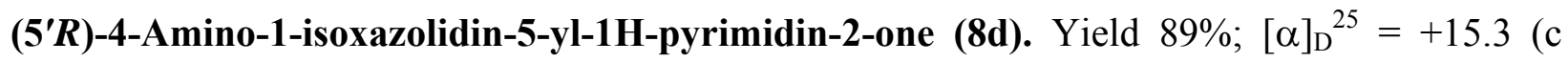
0.55; $\mathrm{MeOH}$ ); m.p. $190-194{ }^{\circ} \mathrm{C}$, amorphous solid (Anal. Calcd. for $\mathrm{C}_{7} \mathrm{H}_{10} \mathrm{~N}_{4} \mathrm{O}_{2}$ : C, 46.15; $\mathrm{H}$, 5.53; N, 30.75\%. Found: C, 46.92; H, 5.49; N, 30.12\%); $\delta_{\mathrm{H}}\left(\mathrm{CD}_{3} \mathrm{OD}, 200 \mathrm{MHz}\right): 2.42-2.52(\mathrm{~m}$, $\left.1 \mathrm{H}, \mathrm{H}_{4^{\prime} \mathrm{a}}\right), 2.56-2.73\left(\mathrm{~m}, 1 \mathrm{H}, \mathrm{H}_{4^{\prime} \mathrm{b}}\right), 3.19-3.30\left(\mathrm{~d}, 1 \mathrm{H}, J=7.4 \mathrm{~Hz}, \mathrm{H}_{5}\right), 5.97$ (m, 1H, $\left.\mathrm{H}_{5^{\prime}}\right), 7.63$ (d, $\left.1 \mathrm{H} J=7.4 \mathrm{~Hz}, \mathrm{H}_{6}\right) ; \delta_{\mathrm{C}}\left(\mathrm{CD}_{3} \mathrm{OD}, 50 \mathrm{MHz}\right): 37.49$, 49.28, 90.84, 95.87, 143.91, 158.38, 167.81 .

\section{Acknowledgements}

We thank the M.I.U.R. and C.N.R. for their partial financial support. 


\section{References}

1. De Clercq, E. Biochim. Biophys. Acta 2002, 1587, 258.

2. De Muys J.-M.; Gordeau, H.; Nguyen-Ba, N.; Taylor, D. L.; Ahmed, P. S.; Mansour, T.; Locas, C.; Richard, N.; Wainberg, M. A.; Rando, R. F. Antimicrob. Agents Chemother. 1999, 43, 1835.

3. (a) Pan, S.; Amankulor, N. M.; Zhao, K. Tetrahedron 1998, 54, 6587. (b) Merino, P.; Franco, S.; Merchan, F. L.; Tejero, T. J. Org. Chem. 2000, 65, 5575. (c) Adams, D. R.; Boyd, A. S. F.; Ferguson, R.; Grierson, D. S.; Monneret, C. Nucleosides Nucleotides 1998, 17, 1053. (d) Fischer, R.; Drucková, A.; Fišera, L.; Rybár, A.; Hametner, C.; Cyrański, M. Synlett 2002, 1113.

4. (a) Leggio, A.; Liguori, A.; Procopio, A.; Siciliano, C; Sindona, G. Tetrahedron Lett. 1996, 37, 1277. (b)Leggio, A.; Liguori, A.; Procopio, A.; Siciliano, C; Sindona, G. Nucleosides Nucleotides 1997, 16, 1515. (c) Colacino, E.; Converso, A.; Liguori, A.; Napoli, A.; Siciliano, C.; Sindona, G. Tetrahedron 2001, 57, 8551. (d) Dalpozzo, R.; De Nino, A.; Maiuolo, L.; Procopio, A.; De Munno, G.; Sindona, G. Tetrahedron 2001, 57, 4035.

5. Chiacchio, U.; Rescifina, A.; Corsaro, A.; Pistarà, V.; Romeo, G.; Romeo, R. Tetrahedron: Asymm. 2000, 11, 2045.

6. (a) Chiacchio, U.; Corsaro, A.; Gumina, G.; Rescifina, A.; Iannazzo, D.; Piperno, A.; Romeo, G. Romeo, R. J. Org. Chem. 1999, 64, 9321. (b) Chiacchio, U.; Corsaro, A.; Iannazzo, D.; Piperno, A.; Rescifina, A.; Romeo, R.; Romeo, G. Tetrahedron Lett. 2001, 42, 1777. (c) Chiacchio, U.; Corsaro, A.; Iannazzo, D.; Piperno, A.; Procopio, A.; Rescifina, A.; Romeo, G.; Romeo, R. Eur. J. Org. Chem. 2001, 1893. (d) Iannazzo, D; Piperno, A.; Pistarà, V.; Rescifina, A.; Romeo, R. Tetrahedron 2002, 58, 581. (e) Chiacchio, U.; Corsaro, A.; Pistarà, V.; Rescifina, A.; Iannazzo, D.; Piperno, A.; Romeo, G.; Romeo, R.; Grassi, G. Eur. J. Org. Chem. 2002, 1206.

7. Stewart, J. J. P. J. Comput. Chem. 1989, 10, 209 and 221.

8. (a) Tronchet, J. M. J.; Iznaden, M.; Barlat-Rey, F.; Dhimane, H.; Ricca, A.; Balzarini, J.; De Clercq, E. Eur. J. Med. Chem. 1992, 27, 555. (b) Tronchet, J. M. J.; Iznaden, M.; BarbalatRey, F.; Komaromi, I.; Dolatshahi, N.; Bernardinelli, G. Nucleosides Nucleotides 1995, 14, 1737. 